Referencia para citar este artículo: Molina-Chávez, W. M. \& Oliva-Figueroa, I. G. (2015). Interfaces complejas en políticas educativas y de juventud: sentidos e identidades. Revista Latinoamericana de Ciencias Sociales, Niñez y Juventud, 13 (2), pp. 1125-1140.

\title{
Interfaces complejas en políticas educativas y de juventud: sentidos e identidades*
}

\author{
Walter Manuel Molina-CHÁVEZ \\ Profesor Universidad de Magallanes, Chile. \\ IVAN GABRIEL OLIVA-FIGUEROA *** \\ Profesor Universidad Austral de Chile, Chile.
}

\begin{abstract}
Articulo recibido en noviembre 6 de 2013; artículo aceptado en enero 13 de 2014 (Eds.)
\end{abstract}
- Resumen (Analítico) En el artículo damos cuenta de las transformaciones e inflexiones socioeconómicas, sociopolíticas y socioculturales - a escala global-de las sociedades contemporáneas, en correlato con los procesos situados de configuración de sentidos educativos e identidad de las jóvenes y los jóvenes escolares secundarios de Chile. Este estudio lo realizamos en cuatro escuelas secundarias públicas en Santiago de Chile. La metodología es de tipo cualitativa y está basada en 32 entrevistas en profundidad. Los resultados evidencian que en la escuela secundaria se configura una pluralidad de sentidos educativos y emergen nuevas identidades entre las personas jóvenes que la habitan cotidianamente. Concluimos que las actuales transformaciones estructurales y subjetivas en curso, tienen profundas implicancias para el diseño de políticas públicas educativas y de juventud en un contexto de fragmentación social y segregación de las instituciones educativas.

Palabras clave: identidad, escuela secundaria, sentidos educativos, Chile (Tesauro de Ciencias sociales de la Unesco).

Palabras clave autores: políticas educativas, políticas de juventud, interfaces complejas.

\section{Complex interfaces in educational and juvenile politics: meaning and identities in Chile's secondary school system}

- Abstract (analytical): This article describes socio-economic, socio-political and socio-cultural transformations and inflections at a global scale of contemporary societies that are related to the educational processes and identity configuration of young Chilean students in secondary education. The study uses a qualitative methodology and is based on 32 in-depth interviews. Results show that in secondary schools, multiple meanings around education exist and new identities emerge among young people that attend school on a daily basis. The study concludes that current and ongoing structural and subjective transformations do have profound implications for the design of educational policies in contexts involving social fragmentation and segregation of educational institutions.

Este artículo de investigación científica y tecnológica forma parte del proyecto “Juventudes. Transformaciones socioeconómicas, sociopolíticas, y socioculturales de las y los jóvenes en el Chile contemporáneo”. Programa de Investigación Asociativa Comisión Nacional de Ciencia y Tecnología (Conicyt) No SOC1108 - 2012-2015. Mes y año de inicio y finalización de la investigación, enero de 2013 y terminó en octubre de 2013. Área: ciencias de la educación; subárea: educación general.

** Doctor en Ciencias de la Educación, por la Pontificia Universidad Católica de Chile. Profesor Universidad de Magallanes, Departamento de Ciencias Sociales, Carrera de Trabajo Social, Casilla 113-D, Punta Arenas, Chile. Correo electrónico: walter.molina@umag.cl

*** Doctor en Ciencias de la Educación, por la Pontificia Universidad Católica de Chile. Profesor Universidad Austral de Chile. Instituto de Filosofía y Estudios Educacionales. Valdivia, Chile. Correo electrónico: ivanoliva@uach.cl 
Key words: identity, secondary school, educational senses, Chile (Unesco Social Sciences Thesaurus).

Authors key words: educational policies, youth policies, complex interfaces.

\section{Interfaces complexas em políticas educativas e de juventude: sentidos e identidades em escolas de ensino médio no Chile}

- Resumo (Analítico): O artigo reporta as transformações e inflexões socioeconômicas, sociopolíticas e socioculturais à escala global das sociedades contemporâneas, em correlato com os processos situados de configuração de sentidos educativos e identidade dos/as jovens escolares do ensino médio chileno. O estudo foi realizado em quatro escolas públicas de ensino médio situadas em Santiago do Chile. A metodologia é do tipo qualitativa e baseada em 32 entrevistas em profundidade. Os resultados evidenciam que no ensino médio se configura uma pluralidade de sentidos educativos e emergem novas identidades entre os/as jovens que a habitam cotidianamente. Concluiu-se que as atuais transformações estruturais e subjetivas em curso têm profundas implicações para o planejamento de políticas públicas educativas e de juventude em um contexto de fragmentação social e segregação das instituições educativas.

Palavras-chave: identidade, ensino médio, sentidos educativos, Chile (Tesauro de Ciências Sociais da Unesco).

Palavras-chaves autores: políticas educativas, políticas de juventude, interfaces complexas.

-1. Antecedentes generales. -2. Políticas educativas en Chile y políticas de juventud: Interfaces complejas y multidimensionales. -3. Sentidos educativos y procesos de constitución de sujetos desde la escuela secundaria en Chile. -4. Resultados de investigación. -5. Conclusiones. -Lista de referencias.

\section{Antecedentes Generales}

Caminito al costado del mundo, por ahi he de andar,

Buscándome un rumbo, ser socio de esta sociedad, me puede matar. La Renga

Las sociedades latinoamericanas en general y la sociedad chilena en particular, cursan transformaciones globales que han reconfigurado los tradicionales patrones de relaciones entre sujetos e instituciones sociales, mediando hologramáticamente ${ }^{1}$ el tránsito entre instancias locales y globales, a la vez de dar expresión a interfaces complejas que conjugan paradojalmente, emergentes y constrictivos espacios de participación e integración social.

1 Por mediación hologramática entenderemos todo proceso de mutua influencia entre instancias locales y globales de un sistema social. En estos términos, en un sistema complejo toda localidad lleva inscrita la huella matricial de la globalidad a la que pertenece, y a la vez, toda globalidad es influenciada y constantemente actualizada por los comportamientos locales de sus componentes, dando origen a interfaces sociales complejas.
Las formas de participación de las jóvenes y los jóvenes en este contexto, han asumido gravitantes procesos de autonomía, posibilitados por pautas de comunicación autoorganizativas, estructuras descentralizadas, y la mediación fundamental de diversos soportes tecnológicos. Todo ello nos permite postular a escala global-local, una modificación profunda en las matrices comunicativas desde donde se construyen los sentidos educativos, los procesos de identidad juvenil y su correlato con las formas de participación política y social.

Desde esta base, asumimos que toda política pública educativa es ipso facto una política de juventud, y por ello le es irrenunciable abordar la pregunta por la noción de sujeto desde un enfoque generacional, y simultáneamente reconocer, valorar y comprender los procesos de construcción de identidad de los jóvenes y las jóvenes que oficiarán como destinarios de sus lineamientos. En este contexto, sostenemos que es en el amplio registro de las políticas públicas educativas, donde se expresan con mayor radicalidad y profundidad todas las dimensiones epistemológicas subyacentes a lo 
que podemos denominar políticas de juventud de una sociedad.

De este modo, los sentidos y procesos de construcción de identidad de jóvenes en espacios escolares, pueden comportar elementos centralizadores y emergentes de las prácticas subjetivas de participación política en el ámbito educativo en específico, y en el campo de "lo social" en general. Pensar la educación, y particularmente los procesos de escolarización secundaria y la constitución de actores sociales juveniles, se torna en este punto un fenómeno dialógico que expresa todas sus dimensiones de complejidad en la pregunta por los sentidos de la educación de las jóvenes y los jóvenes secundarios en Chile.

Desde esta perspectiva de entendimiento, es posible observar en los actuales procesos de cambios educativos la manifestación de fuertes tensiones entre los fenómenos de globalización y los procesos de construcción de identidad social y cultural locales. A partir de ello, adquiere relevancia explorar en estos vastos y diversos territorios cómo los actores pertenecientes a la educación media, desde sus procesos de construcción de identidad, configuran multidimensionalmente los sentidos en torno a la educación. En consecuencia, sostenemos que la emergencia de configuraciones de sentidos y subjetividades sobre la educación secundaria pública en Chile, se expresa en nuevos sentidos que se constituyen en elementos mediadores entre la experiencia escolar de los sujetos estudiantes y las políticas educativas implementadas -a escala global y local-; obstaculizando o facilitando con ello el logro de una educación secundaria con alta relevancia personal -y social-, calidad académica y mayores grados de equidad tanto en el acceso como en la distribución social de sus resultados de aprendizaje.

\section{Políticas educativas en Chile y políticas de juventud: Interfaces complejas y multidimensionales}

En Chile -en correlato con lo anterior-, es posible describir una acelerada expansión de la matrícula escolar en todos los niveles, pero muy especialmente en el nivel secundario. A modo de ejemplo, mientras la matrícula de enseñanza secundaria en los años 60 alcanzaba un 14\% de cobertura entre las personas de 15 a 19 años, en la actualidad constituye un sistema de acceso masivo con una cobertura cercana a $81 \%$ (Mineduc, 2010, Injuv, 2012). Es decir, ocho de cada diez jóvenes entre se encuentra inserto en alguna modalidad de educación secundaria. Según datos recientes del Socio-Economic Database for Latin American and The Caribbean (Sedlac, 2013), las jóvenes y los jóvenes de Chile, de edades entre 21 y 30 años tienen, en promedio, 12.5 años de estudios, sobrepasando en dos años la media de América Latina y el Caribe. Sin embargo, esta amplia cobertura educacional y sus altos niveles de escolaridad promedio no han estado ligadas a altos niveles de calidad en la prestación de los servicios educativos, lo cual ha generado un conjunto de complejas inequidades e ineficiencias del sistema y una pérdida progresiva de su eficacia externa como mecanismo de movilidad social ascendente (Ocde, 2004, Mineduc, 2010). A modo de ejemplo, la tasa de prevalencia de la deserción escolar en la educación secundaria chilena es de 9,5\% para el grupo socioeducativo de 15 a 19 años de edad, y de 16\% para el grupo de 20 a 24 años (Mineduc, 2013).

Lo anterior demanda nuevos desafíos tanto a las políticas educativas como a las políticas de juventud, y sobre todo implica fortalecer integralmente la educación pública chilena logrando, por una parte, cobertura, permanencia y conclusión de una escolarización secundaria con altos niveles de calidad tanto en sus procesos internos como en sus resultados de aprendizajes al finalizar dicho ciclo educativo; por otro lado, exigiendo que los Estados en la presente coyuntura diseñen políticas públicas robustas que aseguren que estos crecientes niveles de escolaridad de las jóvenes y los jóvenes impliquen oportunidades efectivas de inserción laboral y social postsecundaria. Por último, se requiere que las políticas educativas y de juventudes pongan en su centro la configuración de una ciudadanía juvenil capaz de desplegarse de manera autónoma e incidir sustantivamente en el espacio público, tanto individual como colectivamente, ya sea 
participando a través de los canales abiertos por un régimen político-democrático formal, o mediante su expresión en movimientos sociales juveniles autónomos y diversos, constituidos en los diferentes pliegues de la vida social contemporánea.

En este contexto de análisis, cabe señalar que Sisto y Fardella (2011) sostienen que las políticas educativas implementadas en los últimos años en Chile han estado guiadas por una retórica que él denomina "epocalismo", las que harían referencia a lo siguiente:

"[...] refiere a un esquema justificatorio que establece dualidades y oposiciones entre un pasado y un presente, remarcando la oposición y discontinuidad. Mediante el desarrollo de descripciones factuales de la realidad, se refuerzan estas diferencias, lo que sirve para argumentar orientaciones altamente prescriptivas para sujetos, organizaciones y comunidades[...] En el discurso epocalista, el 'cambio' es presentado como homogéneo, instalando a objetos, dispositivos y personas en una misma dimensión, en un mismo tipo de proceso. De modo tal que el cambio en política pública se presenta, más que como el resultado de opciones políticas, como una consecuencia inevitable de sucesos imperativos, pero abstractos y deslocalizados, tales como la revolución de la información, la globalización, entre otros (Sisto \& Fardella, 2011, p. 125).

A partir del análisis anterior, se evidencia que en el centro del discurso sobre las nuevas políticas públicas en educación, se coloca retóricamente la necesidad de introducir cambios frente a una realidad factual e inevitable, la cual estaría marcada por fenómenos característicos de la época actual como la globalización y la presencia creciente de las tecnologías de la información y las comunicaciones (TIC). Derivado de lo anterior, estos cambios imperativos deben estar orientados normativamente a modificar tanto los modos de gestión de las escuelas, como los referentes identitarios de los profesores y profesoras, para que sus actuaciones sean más eficientes y eficaces, de acuerdo con un paradigma vinculante que Sisto y Fardella (2011, p. 129) denomina "Nuevo Management Público" -NMP-, basado en el cumplimento de estándares de desempeño, incentivos económicos y desempeño individual de los maestros y maestras.

Desde una perspectiva más amplia, diversos autores y autoras de Latinoamérica (Tenti, 2000, Braslavsky, 2001, Berger, 2002, Kessler, 2002, Del Valle, 2010, Molina-Chávez, 2013b) han sostenido que la rápida expansión de la Enseñanza Secundaria en América Latina, y muy particularmente en Chile, ha llegado a una encrucijada que se relaciona con tres fenómenos emergentes y fuertemente interconectados: a) un proceso de masificación y obligatoriedad de la educación primaria y secundaria, con bajos estándares de calidad; b) la creciente tendencia a la segregación social de la oferta educativa, tanto del nivel primario como de los niveles secundario y terciario; y c) la falta de protagonismo y participación de las jóvenes y los jóvenes en los procesos de escolarización secundaria en nuestro continente.

En el caso de Chile, la configuración en los últimos cuarenta años de una estructura de segregación socioeducativa, se expresa en la existencia de escuelas diferenciadas para estudiantes de bajos, medianos y altos ingresos económicos, quienes respectivamente estudian en establecimientos público-municipales, particulares subvencionados, y privados. Por ello, y considerando que los estudiantes y las estudiantes provenientes de familias de menores ingresos se concentran en los colegios municipalizados y/o subvencionados por el Estado, es de alto interés investigativo profundizar en el estudio de los sentidos y subjetividades emergentes desde la propia experiencia escolar de los estudiantes secundarios de este segmento educativo, por cuanto son ellos quienes en el torbellino de los procesos de globalización y neoliberalismo se las han arreglado para producir subjetividades y nuevos sentidos en torno a los procesos de escolarización obligatoria (Frigerio, 2002, Macedo \& Katzkowicz, 2002, MolinaChávez, 2008, 2013a y 2013b). Son estos procesos autónomos los que finalmente inciden decisivamente en sus trayectorias personales/ sociales, en ámbitos clave de la experiencia/ condición juvenil contemporánea, tanto 
en Chile como en América Latina. Dichos ámbitos han sido caracterizados por diversos organismos (Injuv, 2012) a partir de cinco transiciones que implicaría la condición juvenil contemporánea: el término de la educación formal, la incorporación al mundo laboral, la formación de una familia, el cuidado de la salud y el ejercicio de la ciudadanía. Lo anterior, junto con mostrar los principales hitos socio-biográficos en las trayectorias de vida de las jóvenes y los jóvenes, permite sobre todo visualizar el modo en que las personas jóvenes configuran diferencialmente sus trayectorias sociales y ponen en evidencia los severos procesos de exclusión/estratificación social que los afectan gravemente. A modo de ejemplo, Del Valle (2010, p. 593) sostiene que Argentina, Uruguay, Chile, Brasil y Ecuador, poseen sistemas altamente segmentados de educación primaria con tramos minoritarios de educación privada de alta calidad, un elevado costo no subvencionado por el Estado, y un amplísimo segmento de educación pública con escasez de recursos, bajos niveles de innovación y calidad de los servicios educativos ofrecidos a la población de menores recursos.

En consecuencia, es necesaria una mirada que articule la lectura crítica de las transformaciones socio estructurales -a escala global histórico-institucional- que han tenido lugar en las sociedades latinoamericanas -de las cuales la masificación de la escolarización secundaria es un proceso clave-, y simultáneamente, atender a su significación subjetiva desde la experiencia social local y situada de los estudiantes y las estudiantes. Lograr esta articulación resulta relevante tanto para el replanteamiento de las políticas educativas como para para un nuevo diseño de las políticas de juventud en América Latina y en Chile. Lo anterior permitiría configurar interfaces complejas y multidimensionales, tanto en el diseño como en su implementación, pero sobre todo cartografiar e integrar la subjetividad sobre los procesos de escolarización secundaria desde la propia experiencia escolar de las niñas, niños y jóvenes que habitan cotidianamente el espacio escolar de la educación secundaria pública en nuestro continente.

\section{Sentidos educativos y procesos de constitución de sujetos desde la escuela secundaria en Chile}

\subsection{Políticas educativas y configuración de sentidos educativos desde la experiencia escolar en el Chile contemporáneo}

En un contexto de profundos cambios socioeconómicos, sociopolíticos y socioculturales, el principal desafío de la escuela secundaria chilena es orientarse explícitamente hacia la construcción de un sujeto comprometido con los principios de ciudadanía, orientado a la comunicación intercultural y concernida en la gestión democrática de la sociedad. Desde la perspectiva de análisis de Touraine (1997), la escuela debe orientarse a unir los dos mundos que tensionan a los sujetos jóvenes: el mundo que define y condiciona las posibilidades materiales que ofrece la sociedad y el mercado del trabajo, y el universo simbólico que constituye la cultura de la gente joven, y que se difunde desde los medios de comunicación de masas y es transmitida cotidianamente por los pares. De este modo, una vez más se plantea desde el seno de la escuela la permanente tensión entre subjetividad y racionalización, o como alternativamente señala Hopenhayn (2005, p. 45), entre < ratio dura> y "sentido", o en su defecto, entre las diversas variantes de esta misma oposición: tecnología vs cultura; identidad vs medios de comunicación.

En este contexto, algunos autores (Tenti, 2000, Molina-Chávez, 2008) han identificado diversas configuraciones de los sentidos educativos de la escuela pública en América Latina y particularmente en Chile. Para el caso chileno, Molina-Chávez (2008) describe cuatro configuraciones de sentido de la escuela secundaria desde la propia experiencia escolar de los estudiantes: (1) <prepararte para el futuro $>$ (2) < desarrollarte como persona $>$ (3) $<$ tener más cultura $>$ y $(4)<$ vivir la experiencia/ etapa $>$. En este contexto, los sentidos educativos y sociales que los jóvenes y las jóvenes construyen en torno a los procesos de escolarización secundaria, cristalizan en determinadas representaciones sociales, las que pueden estar mediando su propia experiencia escolar al interior de los establecimientos a los 
que asisten cotidianamente, obstaculizando o facilitando con ello el logro de una enseñanza media con alta relevancia social -y personal-, con alta calidad académica y con resultados de aprendizaje que contribuyan significativamente a una inserción de los individuos jóvenes en los diferentes planos de la vida social.

Por otra parte, esta diversidad de configuraciones de sentido identificadas se relaciona análogamente con lo que Kessler (2002) ha denominado una experiencia escolar fragmentada en la escuela secundaria argentina; es decir, la existencia al interior de un mismo nivel educativo de diversos modos de vivir y experimentar los procesos de escolarización obligatoria. Más concretamente, y en los términos planteados por Tenti (2000), para el caso de la escuela secundaria argentina se ha identificado tres orientaciones de sentido principales: a) la escuela secundaria como obligación social -y jurídica-; b) el sentido de la escolarización como posibilidad estratégicainstrumental de acceder a mejores condiciones de vida o inserción laboral post-secundaria; y c) el sentido como amor al conocimiento y al aprendizaje. Al respecto, el mismo autor señala lo siguiente:

Estas tres disposiciones ideal típicas descritas por la literatura no están aleatoriamente distribuidas en la población, sino que por el contrario son el producto de un conjunto de factores interrelacionados. Entre ellos, cabe mencionar los factores culturales, las trayectorias individuales y el lugar que ocupan las personas en la estructura social (Tenti, 2000, p. 10).

Como se puede observar, los sentidos educativos de la educación secundaria tanto en América Latina como en Chile, tienden a una pluralidad de expresiones y se estructurarían en torno a representaciones sociales y a ejes de significados comunes. Por una parte, están aquellos sentidos educativos instrumentales -"prepararte para el futuro"-; por otra, aquellas configuraciones de sentido más orientadas a valores personales o sociales -"desarrollarte como persona"-, o alternativamente, como señala Tenti (2000), están aquellos estudiantes que aún conciben el sentido de la escuela secundaria como "amor al conocimiento".

En este contexto de análisis, Weinstein (2002) señala que la educación puede constituir un canal de integración social de primer orden, y su acción e impacto beneficiaría significativamente tanto a los individuos en su desarrollo personal, como a la sociedad en sus necesidades funcionales, como lo es lograr mayores niveles de integración y cohesión social. Sin embargo, la educación concebida como canal de integración social no opera automáticamente, ni es una realidad inherente a los sistemas educativos. Desde esta perspectiva, las políticas públicas educativas pueden contribuir significativamente a la integración -y cohesión- social, a condición de que reconozcan que integrarse no es solo acceder y disfrutar de oportunidades individualmente, sino que es necesario que en la base de estas políticas exista una orientación o dirección explícita, es decir, un sentido colectivo compartido en el marco de una sociedad determinada.

Al respecto, y desde una perspectiva más global, Sobrero (2011) sostiene que al analizar las configuraciones de sentido en torno a la educación pública en Chile en los discursos de la política educativa, implementada en el periodo 1990-2007, se puede observar que se han producido tanto continuidades como desplazamientos de sentido en torno a la educación pública, lo cual ha dado origen a "un conjunto complejo y heterogéneo de interacciones que configuran relaciones de poder en el que se encuentran y desencuentran relatos y visiones diversas" (Sobrero, 2011, p. 115). La autora concluye que desde las políticas educativas se promueven sentidos que sugieren un tratamiento indiferenciado entre la esfera pública y la esfera privada, a partir de lo cual la educación pública quedaría subsumida a una "racionalidad economicista" que restringe su campo de acción a la gestión de los recursos insumos internos del sistema educativo; lo que enmarca fuertemente la diversidad de experiencias subjetivas de los actores educativos, tanto dentro como fuera del espacio escolar. 


\subsection{Sujetos, subjetividades e identidades}

En los actuales debates en ciencias sociales y humanidades han resurgido con fuerza las nociones de sujeto, identidad y subjetividad como elementos articuladores de diversas reflexiones que intentan dar cuenta de las emergentes condiciones socioeconómicas, sociopolíticas y socioculturales en el mundo contemporáneo. Desde el campo de la sociología de la educación, estos debates han tomado la forma de una profunda tensión entre los componentes sistémicos -estructurales- de los procesos de escolarización y los modos como los niños, niñas y jóvenes despliegan su subjetividad en el espacio escolar contemporáneo. En un marco de análisis más general, y específicamente a partir de la modernidad, se han producido múltiples desplazamientos en el desarrollo conceptual de las categorías utilizadas para describir la emergencia del sujeto moderno.

Para Payne (2002), los orígenes del concepto de sujeto son opacos; pero aún así, es posible rastrear la noción de sujeto hasta Kant, o incluso Descartes. En ambos filósofos, es posible entrever una fuerte reacción contra el privilegio del yo, del sí mismo o simplemente de la idea de individuo que emergía utópicamente en el pensamiento humanista. Para este mismo autor, "el concepto de sujeto es en gran medida producto de la obra de Lacan, para quien los sujetos son radicalmente distintos del yo, que es concebido como un producto ilusorio del estadio del espejo" (Payne, 2002, p. 600). Por otra parte, y más tardíamente, es Althusser quien lo emplea como clave para analizar la transformación de los individuos humanos en sujetos, gracias a los efectos imaginarios de la ideología -capitalista y liberal moderna-. Como se puede observar, la tendencia general en estos debates es concebir al sujeto como "efecto" o "reflejo" de una estructura o un sistema -educativo en nuestro análisis-, y no como fuente primaria de construcción de esta. En términos sintéticos, en estas concepciones el sujeto no habla y no es(tá) en el origen del sentido; por el contrario, es hablado por la ley y la cultura, ya sea para afirmarlo o negarlo/invisibilizarlo.

Por otra parte, y desde una perspectiva filosófica latinoamericana, Acosta (2011) señala que las conceptualizaciones modernas sobre el sujeto se han expresado paradigmáticamente de diversas formas en el plano discursivo: sea en el modo de sujeto sustancial (Descartes), sea en el de sujeto epistemológico-formal (Kant), sea como sujeto en el histórico-ontológico (Hegel) o en el histórico-estructural (Marx). Desde otro ángulo, la perspectiva deconstructivista -posmoderna y postestructuralista- ha declarado en reiteradas oportunidades la muerte del sujeto -moderno-, tematizando sobre su proceso de fragmentación y la emergencia omnipresente de mecanismos sistémicos de mediación de "lo social", como lo sería el Mercado, y que invisibilizan al sujeto factico-moderno. Es decir, estaríamos frente a la re-emergencia del sujeto abstracto, universal y metafísico - "espíritu absoluto", "ego cogito", "pueblo", "Estado", "mercado total", etc.-, que fue visibilizado desde la modernidad y frente a lo cual la filosofía latinoamericana del sujeto (Zemelman, 1998, Hinkelammert, 2003, Acosta, 2011) reivindica y afirma la presencia de un sujeto empírico $e$ histórico concreto que se materializa tanto en su dimensión individual como colectiva/social.

El reconocimiento de estos órdenes de complejidad fenomenológica, lleva al pensamiento crítico latinoamericano a poner en tela de juicio las nociones de sujeto concebidas en el marco del pensamiento tanto moderno como posmoderno, enunciando desde otros parámetros categoriales el surgimiento de un "sujeto vivo" capaz de enfrentar, autoorganizar y trascender la inercia de un sistema que lo condiciona fuertemente y muchas veces lo "ahoga".

Desde una perspectiva lingüística, Benveniste (1971) destaca que la subjetividad no es otra cosa que la capacidad del locutor para presentarse como sujeto, y por ello, sería el lenguaje donde deben ir a buscarse los fundamentos de esta aptitud-capacidad. Al respecto señala lo siguiente:

Es en y por el lenguaje como el hombre se constituye como sujeto (...) para conseguirlo, 
se apropia de ciertas formas que la lengua pone con este fin a su disposición, y en primer lugar del pronombre Yo, cuyo uso es el fundamento mismo de la conciencia de sí. (...) La conciencia de sí no es posible más que experimentándose por contraste. Yo no empleo "yo" sino dirigiéndome a alguien, que será en mi alocución un tú: no hay subjetividad sin intersubjetividad (Benveniste, 1971, p. 179).

Esta dimensión lingüística y auto-ecoorganizacional de la subjetividad, es a nuestro juicio una de las claves para avanzar en una comprensión compleja de los sujetos sociales y específicamente su despliegue y emergencia en el campo socioeducativo. Ello permite hablar desde la experiencia, en tanto soporte, que cristaliza en lo subjetivo. Así, la subjetividad emerge como lo individual, lo propio, lo diferente del otro, pero inserto en la trama de lo socio/histórico.

En este contexto, para Martínez y Neira (2009) una misma experiencia vivida por personas diferentes adquiere valoraciones únicas en cada caso. De este modo, el interés renovado de las ciencias sociales por la subjetividad no está orientado a re-posicionar al "sujeto cartesiano" -racional-, en tanto sujeto cognoscente referenciado unidimensionalmente en torno al conocimiento; el nuevo interés del pensamiento social está puesto en el sujeto de la experiencia, entendiendo esta como la apertura del propio sujeto a la "otredad", a la alteridad y a los modos específicos de ser y estar en el mundo, es decir, los <modos de subjetivación $>$. No obstante, la posibilidad de ser sujeto de la propia experiencia, está mediada por las interfaces entre dos planos o dimensiones de oscilación en los sujetos concretos: a) su dependencia, control y ligazón con el "otro"; y b) la sujeción a su propia identidad expresada en sus prácticas sociales y el conocimiento de sí. Desde esta base, el sujeto deviene en dinámicas semiotizadas auto-eco-organizadas que pueden modificarse a sí mismas o que pueden ser modificadas desde sus relaciones externas y que, por lo tanto, entran en interacción con el medio que rodea al individuo en sus circunstancias.

En esta línea de reflexión nos parece muy relevante la pregunta que se hacen Martínez y Neira (2009, p. 19) en el marco de este horizonte comprensivo de la subjetividad en el mundo contemporáneo: ¿cuáles son los mundos plegados en sus esencias que constituyen nuestra subjetividad en la actualidad? Y más específicamente para nosotros en el contexto de este artículo: ¿cuáles son esos mundos plegados en el campo educativo y/o en el espacio escolar secundario?

En este marco de análisis, para diversos autores y autoras (Castell, 2003, Larraín, 2011, Molina-Chávez, 2011) el proceso de configuración de identidades personales -y sociales- se expresa en una dialéctica irreductible de interacción entre sujeto y sociedad. Es decir, hablar de identidad hace referencia directa a un proceso de construcción social que va más allá del propio individuo, pero que tiene un vínculo de implicación mutuo con él: el individuo es, tal vez, el punto de enunciación del proceso de construcción de su propia identidad, pero también lo es la sociedad en la que está inserto. Es en estas coordenadas de similitud/diferencia que los sujetos y los grupos sociales configuran sus propias fronteras y horizontes identitarios. Desde esta perspectiva, la identidad es un proceso de construcción histórico-social, pues desde esa historicidad los sujetos dan forma a sus aspiraciones, a sus sueños, a sus desafíos, y los proyectan colectivamente hacia las nuevas generaciones.-

Simultáneamente y desde una perspectiva sociológica, Castells (2003) sostiene que en el proceso de construcción de las identidades se utilizan materiales de la historia, la geografía, la biología, las instituciones productivas y reproductivas, y la memoria colectiva, entre otros aspectos relevantes a considerar. Sin embargo, se destaca que los individuos, grupos sociales y sociedades, procesan dichos materiales, reordenando su sentido de acuerdo con las condiciones sociales y los proyectos culturales vigentes en una estructura social concreta. A modo de hipótesis generativa, Castell (2003, p. 29) plantea que, dado que la construcción social de la identidad generalmente tiene lugar en un contexto marcado por las relaciones de poder, 
existirían tres variantes de la construcción de las identidades: a) identidad legitimadora b) identidad de resistencia c) identidad proyecto. La primera es introducida por las instituciones dominantes en la sociedad para extender y racionalizar su dominación frente a los actores sociales. La segunda es generada por aquellos actores sociales que se encuentran en posiciones/ condiciones devaluadas o estigmatizadas socialmente, por lo cual construyen trincheras de resistencia y supervivencia basándose en principios diferentes $\mathrm{u}$ opuestos a los que imperan en las instituciones de la sociedad. Y finalmente, la identidad proyecto surge cuando los actores sociales, basándose en los materiales culturales de los que disponen, construyen una nueva identidad que redefine su posición en la sociedad, y se orientan a transformar la estructura social en su conjunto.

A modo de síntesis, este mismo autor destaca que aquellas identidades que comienzan como resistencia pueden inducir proyectos $\mathrm{y}$, también, con el devenir del tiempo, estas pueden convertirse en dominantes o hegemónicas en las instituciones de la sociedad, con lo cual se vuelven identidades legitimadoras para racionalizar su dominio. En efecto, la dinámica de las identidades a lo largo de la secuencias antes descritas, muestran que, desde el punto de vista de la teoría social, ninguna identidad puede ser una esencia, y ninguna identidad per se es un valor progresista o regresivo fuera de su contexto histórico concreto. Lo anterior es reafirmado por De la Torre $(2002$, p. 77$)$ quien señala que "La identidad es más que nada un concepto relacional, que se gesta en las prácticas intersubjetivas entre los individuos y la sociedad". En la misma línea argumental, López (2011) sostiene que en la base de la idea de identidad se encuentra el proceso mediante el cual un sujeto logra definirse a sí mismo en el marco de una cultura, construyendo de este modo un discurso y realizando unas prácticas cotidianas que le permiten decir "yo soy" de manera unívoca y permanente, a partir de sus fuentes más próximas y localizadas simbólicamente.

\section{Resultados de investigación}

\subsection{Enfoque, material y método de investigación}

En un contexto de diversidad de enfoques metodológicos, se ha optado por un enfoque de tipo cualitativo, lo cual permite indagar sobre los sentidos educativos de los procesos de escolarización secundaria y la configuración de identidad de los estudiantes y las estudiantes, en tanto sujetos educativos y sociales. A continuación describimos los criterios y procedimientos metodológicos utilizados:

\section{a) Casos y sujetos del estudio}

En el contexto de la presente investigación utilizamos el "estudio de casos" (Ottemberger, 2000) para seleccionar tanto las escuelas públicas como los individuos estudiantes entrevistados. En este contexto metodológico, estructuramos la muestra del presente estudio a partir de los siguientes criterios de selección:

- Los sujetos participantes de este estudio fueron 32 estudiantes de $4^{\circ}$ año de enseñanza media. Estos sujetos pertenecen a liceos municipales localizados en cuatro comunas de la Región Metropolitana, Chile.

- Los individuos participantes están distribuidos proporcionalmente entre hombre y mujeres. En cada liceo seleccionamos por orden de lista, y entre los asistentes el día de la entrevista, a cuatro hombres $y$ cuatro mujeres. Escogimos cuatro comunas y respectivamente cuatro liceos $<$ representativos $>$ de diferentes estratos socioeconómicos ${ }^{2}$ :
El grupo socioeconómico al que pertenece el establecimiento sigue la clasificación del Simce: A (bajo), B (Medio- Bajo), C (Medio); D (Medio - Alto y E (Alto). Cabe señalar que no existen establecimientos municipales que atiendan población escolar del estrato socioeconómico alto. 


\begin{tabular}{|c|c|c|c|}
\hline $\begin{array}{c}\text { Estable- } \\
\text { cimiento }\end{array}$ & Comuna & $\begin{array}{c}\text { Grupo } \\
\text { Socio- } \\
\text { económico }\end{array}$ & $\begin{array}{c}\text { Número } \\
\text { Entrevistas }\end{array}$ \\
\hline LICEO 4 & $\begin{array}{c}\text { San } \\
\text { Ramón }\end{array}$ & $\begin{array}{c}\text { A } \\
\text { (Bajo) }\end{array}$ & 8 \\
\hline LICEO 3 & $\begin{array}{c}\text { La } \\
\text { Pintana }\end{array}$ & $\begin{array}{c}\text { B } \\
\text { (Medio Bajo) }\end{array}$ & 8 \\
\hline LICEO 2 & $\begin{array}{c}\text { La } \\
\text { Florida }\end{array}$ & $\begin{array}{c}\text { C } \\
\text { (Medio) }\end{array}$ & 8 \\
\hline LICEO 1 & $\begin{array}{c}\text { Las } \\
\text { Condes }\end{array}$ & $\begin{array}{c}\text { D } \\
\text { (Medio alto) }\end{array}$ & 8 \\
\hline & & & 32 \\
\hline
\end{tabular}

\section{b) Técnicas de producción y análisis de} datos

La principal herramienta de producción de datos fue la entrevista en profundidad, por cuanto esta técnica de investigación social tiene la potencialidad de acceder a los significados que los actores sociales investigados construyen sobre el o los sentido(s) de la enseñanza secundaria pública en Chile.

Por otra parte, analizamos el material textual producido a través de las entrevistas mediante herramientas provenientes de la semántica estructural (Greimas, 1971), enfoque que plantea que la lengua está hecha de oposiciones, por lo tanto de continuidades y discontinuidades. En este contexto, es posible comprender la concepción de estructura que es definida como: "la presencia de dos términos y de la relación entre ellos existente" (Greimas, 1971, p. 28). Esta distinción implica dos consecuencias para el análisis semántico: un solo término-objeto no conlleva significación y la significación presupone la existencia de la relación. Es decir, la condición necesaria de la significación es la aparición de la relación entre dos términos. En el plano lingüístico, a propósito de la relación de disyunción, es posible identificar, por tanto, dos términos que no obstante son opuestos entre sí, poseen un eje semántico en común. Ello permite identificar la doble naturaleza de estas relaciones semánticas entre dos términos: conjunción y disyunción -continuidad y diferencia-. Lo anterior permite a Greimas (1971) identificar tres tipos de estructuras semánticas elementales: a) estructuras paralelas; b) estructuras subordinadas o jerarquizadas; y c) estructuras semánticas cruzadas. El hecho de que los términos o lexemas por sí mismos no conlleven significación, se sustenta en una concepción de lengua en la cual a esta no se la considera como un sistema de signos, sino como una trabazón de estructuras semánticas.

\subsection{Sujetos e identidad en la escuela secundaria pública chilena}

A continuación presentamos un análisis de las principales oposiciones semánticas que emergen en el discurso de los sujetos estudiantes entrevistados; con esta finalidad hemos escogido algunos de los textos producidos. Al respecto, cabe señalar que la primera pregunta que realizamos a los estudiantes y a las estudiantes en el contexto de las entrevistas en profundidad, fue la siguiente: ¿Quién es Andrea? O alternativamente: Si tuvieras que presentarte ante otras personas: ¿Cómo lo harías?

Para el Caso 1 -liceo comuna de Las Condes-, identificamos algunas estructuras semánticas paralelas referidas a la identidad como eje de significados: 

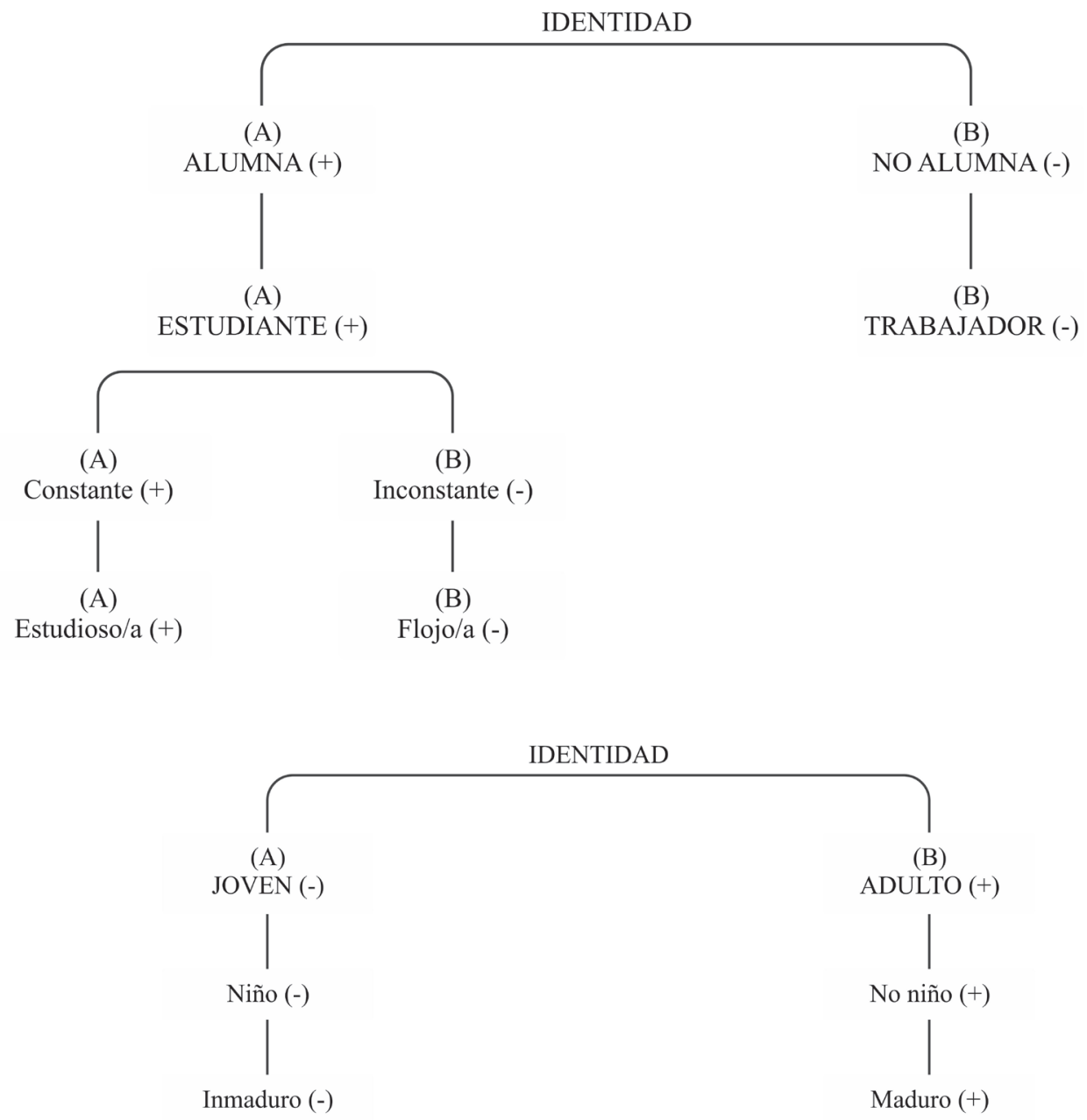

Como es posible observar, desde la experiencia de los jóvenes y las jóvenes estudiantes, emergen estructuras semánticas o de significados que dan cuenta de una identidad desdoblada en dos dimensiones claramente delimitadas. Por una parte, los textos nos refieren al sujeto como alumna o alumno, y alternativamente se definen como estudiantes. La presencia de ambas denominaciones da cuenta del importante lugar de los procesos de escolarización en la configuración de su identidad personal y social. En este marco, el proceso de construcción de identidad se realiza en torno a la dimensión más instrumental de la escuela y de su experiencia escolar, es decir, se trata de una identidad estructurada principalmente en torno a sus deberes escolares y a la necesidad de posicionarse adecuadamente en los procesos de selección y distribución de posiciones o roles sociales que llevan a cabo al interior del espacio escolar.
Por otra parte, emerge el término joven, que remite a una categoría social más amplia y que ubica la construcción de identidad fuera del espacio escolar. A modo de ejemplo, en el texto analizado el término joven se opone semánticamente a adulto. Analíticamente ambas categorías explicitan las siguientes equivalencias: joven $\approx$ niño; joven $\approx$ inmaduro. Inversamente el lexema adulto hace las siguientes relaciones: adulto $\approx$ madurez; adulto $\approx$ no niño. Como se puede inferir en los textos presentados, la adultez es claramente homologada a madurez. Desde este punto de vista, las identidades que construyen los sujetos estudiantes emergen principalmente como respuesta a un desafío de maduración personal y adquisición de responsabilidades vinculadas fuertemente a sus procesos de maduración psicosocial, lo cual es explícitamente demandado por los diversos agentes educativos 
presentes en el espacio escolar secundario.

Para el análisis del segundo caso -liceo de la comuna de La Florida-, fue posible identificar algunas de las siguientes estructuras semánticas paralelas y jerarquizadas, relacionadas con la construcción de la identidad personal y social de los sujetos estudiantes entrevistados:
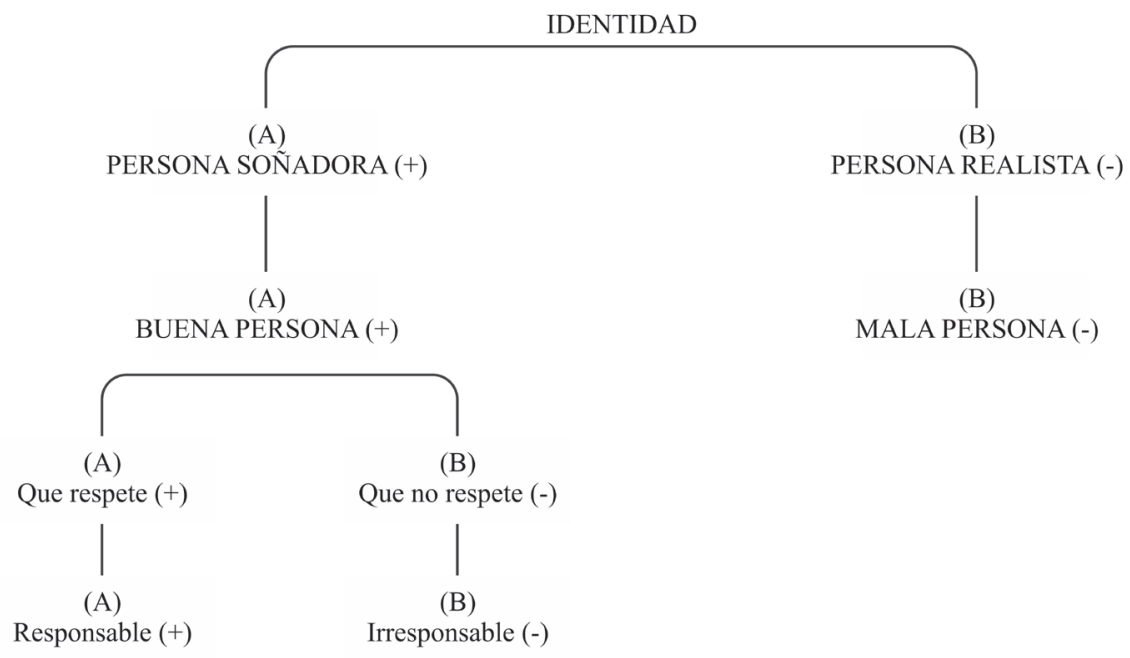

La estructura semántica antes presentada da cuenta de una identidad construida sobre la siguiente oposición: persona soñadora/persona realista. Claramente esta oposición vincula la capacidad de los jóvenes y las jóvenes de imaginar escenarios futuros más allá de las limitaciones y restricciones del entorno escolar o social que los contextualiza. En el marco de esta oposición, algunos de los individuos estudiantes entrevistados construyen una (auto) valoración que se expresa en la aserción $<$ soy una buena persona $>$.

Esta oposición de base da origen a una nueva estructura semántica subordinada, es decir, aquella que surge de un término previamente enunciado -"buena persona"y que en este caso incluye las siguientes oposiciones: que respete/que no respete $\mathrm{y}$ responsable/irresponsable. Dichos términos semánticos indican claramente que la identidad de los sujetos estudiantes de este liceo o escuela pública-municipal en Chile está construida sobre uno de los ejes de sentidos educativos previamente identificados por Molina-Chávez (2008) para la escuela secundaria pública en Chile. Es decir, aquel sentido de la educación secundaria descrito por los jóvenes y las jóvenes que enfatiza los desafíos personales de la experiencia escolar y que se sintetiza en la

expresión $<$ desarrollarse como persona $>$. Como es posible observar, en esta configuración de la identidad personal y social de los individuos estudiantes secundarios en Chile, adquieren una especial relevancia los procesos de maduración psicosocial y la interiorización de valores o normas vinculadas al esfuerzo personal, lo que les permitirá acceder a las metas a las que los sujetos escolarizados aspiran en la vida social. Una estudiante lo expresa de la siguiente manera:

$<$ soy una persona realista, súper realista, pero igual que tiene sus sueños que es tener todo lo que yo quiero, todo lo que me pueda hacer feliz en cuanto a mi vida, tanto en lo personal, en lo de trabajo, lo de familia y todo eso. En lo personal sería llegar a ser una buena persona, responsable y con valores, luego llegar a tener una pareja estable, tener una familia, ¿me entiende? Como que eso sería lo personal. Y llegar a completar mis metas, en el trabajo, en el estudio > (E3, LF, Cecilia, p.2).

De este modo, los individuos estudiantes secundarios entrevistados enfatizan la oposición entre persona soñadora versus persona realista, lo cual se transforma en un eje estructurador transversal en los discursos de los estudiantes 
y las estudiantes de las diversas escuelas secundarias públicas.

Para el análisis del tercer caso -liceo de la comuna de La Pintana-, las entrevistas han permitido identificar algunas de las siguientes estructuras semánticas paralelas y jerarquizadas relacionadas con la identidad personal y social de los sujetos jóvenes estudiantes:

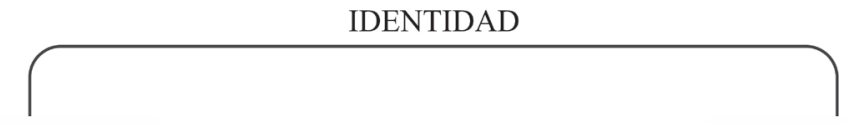

(A)

PERSONA RESPONSABLE (+)

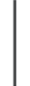

(A)

PERSONA CON METAS $(+)$

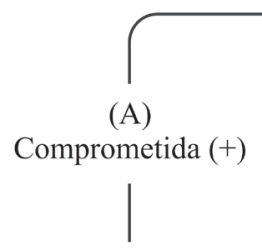

(A)

Esforzada $(+)$
(B)

PERSONA IRRESPONSABLE (-)

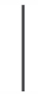

(B)

PERSONA SIN METAS (-)
En los textos analizados emergen unas estructuras semánticas paralelas que dan cuenta de la identidad construida en torno a la responsabilidad y a tres ámbitos específicos de su experiencia: escolar, familiar y social. En la estructura paralela presentada, una persona responsable es simbólicamente una persona con metas y propósitos más concretos, instrumentales $\mathrm{y}$ vinculados a la movilidad económica y social. Un estudiante ejemplifica sus metas de la siguiente manera:

<es poder sacar, porque yo vivo acá en La Pintana, es poder sacar a mi mamá junto con mi hermano de acá donde viven, ser alguien importante, para mí y mi familia. Tener una familia estable, no pasar por lo que yo pasé, ser mejor que mi padre y darle lo mejor a mis hijos. $Y$ meta profesional la verdad es que estoy postulando a la Escuela de Carabineros y quiero ser el mejor. Porque como le dije ya yo ya hice el servicio militar, lo hice cuando estaba en segundo medio que es el nuevo programa> (E7, LP, Alejandro, 3).
Al respecto, es posible identificar diversas fuentes de responsabilidad y esfuerzo personal no solo vinculadas al trabajo académico o al "oficio de ser alumno" (Baeza, 2001), y no restringidas solo a sus aspiraciones individuales vinculadas a la posibilidad de dar continuidad de estudios superiores en el ámbito de la Universidad o de la educación superior. En el texto anteriormente presentado emerge un ámbito de la responsabilidad vinculada al trabajo del sujeto, en pos de configurar unas trayectorias educativas, laborales o sociales que articulen tanto las dimensiones estructurales de base como los esfuerzos personales y despliegues subjetivos de los sujetos, de tal forma que permitan lograr una movilidad social integral de las jóvenes y los jóvenes.

Finalmente, presentamos el cuarto caso de análisis de las valorizaciones referidas a la identidad que construyen estudiantes del liceo municipal de la comuna de San Ramón -estrato socio-económico bajo-. Aquí las estructuras paralelas y subordinadas identificadas son las siguientes: 


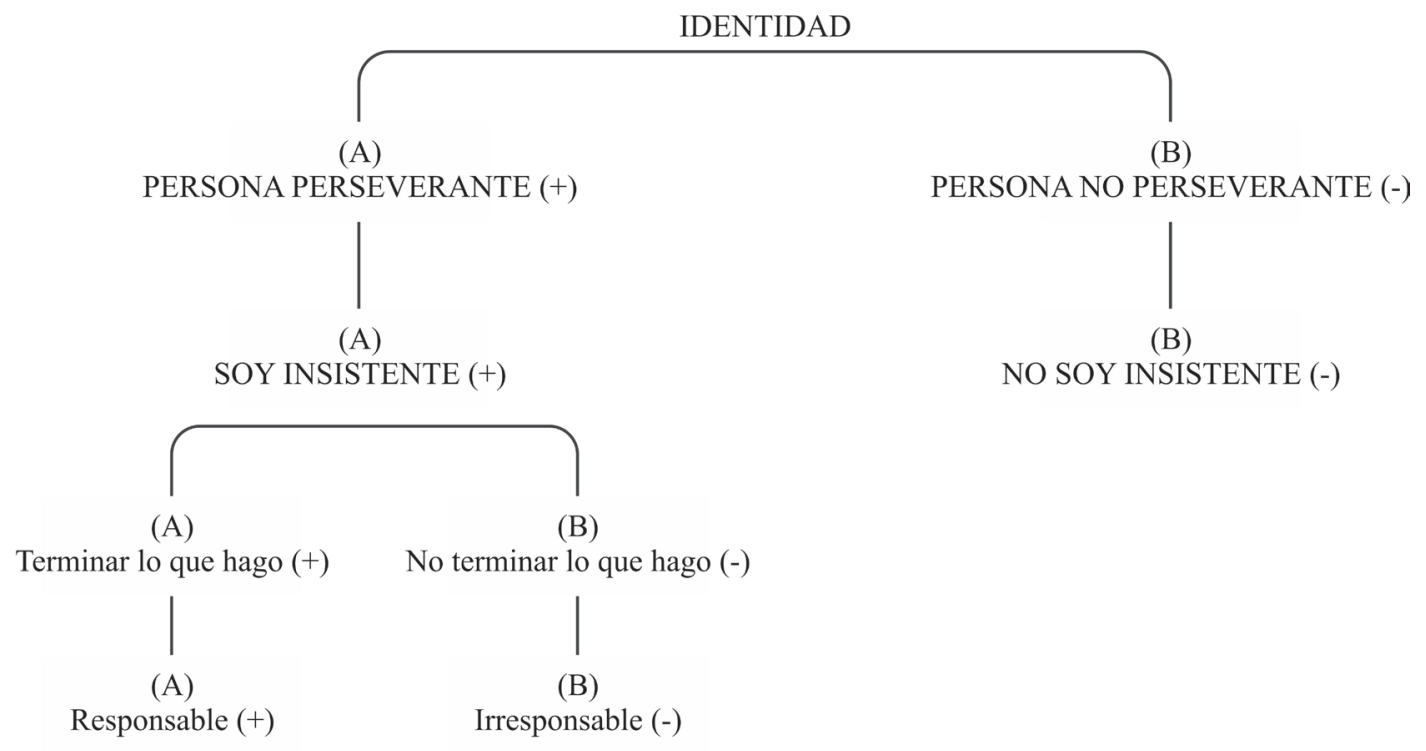

Las estructuras paralelas presentadas están construidas en torno a la oposición entre persona perseverante/persona no perseverante. Los términos de esta oposición nos remiten a la capacidad de los estudiantes y las estudiantes de llevar adelante diferentes tareas y demandas propias de la vida escolar y de la participación social, en el entorno comunitario en el que se ubican las escuelas y liceos público-municipales a los que asisten.

Desde otra perspectiva, un estudiante señala lo siguiente:

$<$ soy un joven bastante activo. Participo en talleres del colegio, soy seleccionado de San Ramón de "Cheearleader". Practico karate (I), trabajo, soy pirata de música, en fin. Aparte de trabajar estudio, y eso. Me mantengo bastante ocupado... Como estudiante no soy el mejor estudiante, es más, no diría que soy buen estudiante> (E6, SR, Danny, p.1-3).

Desde esta base, la configuración identidad de los sujetos jóvenes fluye entre una identidad comprometida en diversas iniciativas comunitarias y actividades extraescolares al interior de las escuelas. Por otra parte, los aspectos identitarios vinculados a su condición de estudiante tienden a ser expresados en términos negativos que destacan las diversas limitaciones que tienen en su desempeño escolar y social cotidiano.

\section{Conclusiones}

Con las reflexiones propuestas esperamos contribuir a resituar en sus dominios de complejidad, la pregunta por las configuraciones de los sentidos y la emergencia de las identidades de los sujetos educativos en el espacio escolar de la escuela secundaria -obligatoria, masiva y segmentada- chilena, en los inicios del Siglo $\mathrm{XXI}$, en una perspectiva que articule miradas hologramáticas tanto globales como locales en torno a los procesos de escolarización secundaria en Chile y en América Latina.

En este contexto, resulta relevante reconocer las dimensiones de complejidad epistémica de los sentidos educativos que emergen desde la experiencia escolar de los sujetos estudiantes que actualmente asisten a la enseñanza secundaria en Chile. A partir de ello, sostenemos que tanto las políticas educativas dirigidas a fortalecer los procesos de escolarización secundaria, como las políticas de juventud dirigidas a promover el acceso de las jóvenes y los jóvenes a diversos derechos sociales, debieran reorientarse y promover acciones -y programas- que permitan integrar la pluralidad de los sentidos educativos y las nuevas identidades emergentes en los sujetos escolarizados, en su rediseño y renovación, en pos de lograr mayores impactos sociales. Evidentemente, dichas acciones se enfrentarán 
con una paradoja central identificada en el discurso de los sujetos estudiantes analizados en la presente investigación, quienes coinciden en que la enseñanza secundaria tiene como principal sentido educativo tanto <prepararte para el futuro $>$ como <desarrollarte como persona $>$. No obstante ello y como hemos revisado anteriormente, los actuales niveles de logro académico amenazan seriamente el cumplimiento de dichos sentidos educativos primarios, lo que es destacado por los sujetos jóvenes estudiantes de escuelas secundarias públicas en Chile. En la perspectiva de superar la complejidad inmanente de esta paradoja, la educación pública tendría entre sus principales desafíos la tarea de (re)construir el carisma y la fuerza movilizadora que tuvo la enseñanza pública en momentos clave del desarrollo de la sociedad chilena.

En síntesis, en el presente trabajo hemos intentado un ejercicio interdisciplinario de entendimiento de las inflexiones e interfaces socioeconómicas, sociopolíticas y socioculturales contemporáneas, en correlato con la pluralidad de sentidos educativos y las configuraciones de identidades de los sujetos educativos al interior del espacio escolar secundario. Las mediaciones sistémicas y huellas hologramáticas que fluyen entre los planos locales y globales donde co-ocurren estos fenómenos son, a nuestro juicio, una de las prospectivas relevantes de una ciencia social compleja y transdisciplinaria en el ámbito de las políticas públicas educativas y de juventud.

\section{Lista de referencias}

Acosta, Y. (2011). La constitución del sujeto en la filosofía latinoamericana. En E. Gruner (coordinador). Nuestra América $y$ el pensar crítico. Fragmentos de Pensamiento Crítico de Latinoamérica $y$ el Caribe, (pp. 75-90). Buenos Aires: Consejo Latinoamericano de Ciencias Sociales.

Baeza, J. (2001). El oficio de ser alumno en jóvenes de liceo de sector popular. Santiago de Chile: Universidad Católica Raúl Silva Henríquez.

Benveniste, E. (1971). Problemas de lingüística general. México, D. F.: Siglo XXI.
Berger, R. (2002). Buscando un nuevo sentido para la educación escolarizada. En Orealc (eds.) ¿Qué educación secundaria para el siglo XXI?, (pp. 73-82). Santiago de Chile: Unesco, Orealc.

Braslavsky, C. (org.) (2001). La educación secundaria, ¿cambio o inmutabilidad? Análisis y debate de procesos europeos y latinoamericanos contemporáneos. Buenos Aires: Iipe, Unesco, Santillana.

Castells, M. (2003). La era de la información. Economía, sociedad y cultura. El poder de la identidad. Madrid: Siglo XXI.

De la Torre, R. (2002). Crisis o revelación de la identidad en la sociedad contemporánea. Nómadas, (16), pp. 76-85. Recuperado el 25 de octubre de 2013, de:

http://dialnet.unirioja.es/servlet/articulo/ pdf.

Del Valle, A. (2010). Productivismo, estratificación y servicios educativos en América Latina. Revista Latinoamericana de Ciencias Sociales, Niñez y Juventud, 8 (1), pp. 577-605.

Frigerio, G. (2002). Entre la repetición y la ocasión de lo nuevo. El derecho a inventar otra cosa. En Orealc (eds.) ¿Qué educación secundaria para el siglo XXI, (pp. 47-71). Santiago de Chile: Unesco, Orealc.

Greimas, A. J. (1971). Semántica estructural. Investigación metodológica. Madrid: Gredos.

Hinkelammert, F. (2003). El sujeto y la ley. El retorno del sujeto reprimido. San José: Euna.

Hopenhayn, M. (2005). Sentidos de la educación. Revista de educación, 317, pp. 32-49.

Injuv (2012). Séptima encuesta nacional de juventud 2012. Santiago de Chile: Instituto Nacional de la Juventud.

Kessler, G. (2002). La experiencia escolar fragmentada. Estudiantes y docentes en la escuela media de Buenos Aires. Buenos Aires: Iipe, Unesco.

Larraín, J. (2011). ¿América Latina moderna? Santiago de Chile: LOM.

López, M. (2011). Producción y expresión de la subjetividad en la juventud contemporánea. Revista de la Facultad 
de Trabajo Social U. P. B., 27 (27), pp. 13-21.

Macedo, B. \& Katzkowicz, R. (2002). Educación secundaria: balance y prospectiva. En Orealc (eds.) ¿Qué educación secundaria para el siglo XXI?, (pp. 123-162). Santiago de Chile: Unesco, Orealc.

Martínez, J. E. \& Neira, F. (2009). Cátedra Lasallista: Miradas sobre la subjetividad. En J. E. Martínez \& F. Neira (compiladores). Miradas sobre la subjetividad, (pp. 13-19). Bogotá, D. C.: Universidad de La Salle.

Ministerio de Educación de Chile (2010). Calidad para Todos, Ministerio de educación, Cuenta Pública 20062010. Santiago de Chile: Ministerio de Educación de Chile.

Ministerio de Educación de Chile (2013). Medición de la deserción escolar en Chile. Santiago de Chile: Ministerio de Educación de Chile.

Molina-Chávez, W. (2008). Sentidos de la enseñanza media desde la experiencia escolar de estudiantes de liceos municipales. Estudios Pedagógicos, 24 (1), pp. 105-122.

Molina-Chávez, W. (2011). Identidad regional en Magallanes, sus expresiones simbólicas y territoriales. Magallania, 39 (1), pp. 59-69.

Molina-Chávez, W. (2013a). Juventudes escolarizadas, sentidos y metáforas sobre el liceo público municipal en el Chile contemporáneo. Revista Última Década, 38, pp. 37-65.

Molina-Chávez, W. (2013b). Sentido de futuro en estudiantes secundarios: paradojas de equidad y calidad desde su experiencia escolar. Revista Estudios Pedagógicos, 34 (1), pp. 143-164.

Ocde (2004). Revisión de politicas nacionales de educación. Chile/París: Organización para la Cooperación y el Desarrollo Económico.

Ottemberger, A. (2000). Estudios de Casos en la Investigación Social. Santiago de Chile: Universidad Tecnológica Metropolitana.

Payne, M. (2002). Diccionario de teoría crítica $y$ estudios culturales. Buenos Aires: Paidós.
Sisto, V. \& Fardella, C. (2011). Nuevas políticas públicas, epocalismo e identidad: el caso de las políticas orientadas a los docentes en Chile. Sorocaba, 37 (1), pp. 121-141.

Sedlac \& The World Bank (2013). SocioEconomic Database for Latin America and the Caribbean. Buenos Aires: Universidad Nacional de La Plata, Banco Mundial. Recuperado el 25 de octubre de 2013, de:

http://sedlac.econo.unlp.edu.ar/esp/ estadisticas.

Sobrero, V. (2011). Configuraciones de sentido en torno a la educación pública en Chile. Análisis de las continuidades y desplazamientos de sentido en torno a la educación pública, en los discursos de la política educativa a partir de 1990. Santiago de Chile: Universidad Católica de Chile.

Tenti, E. (2000). Culturas juveniles y cultura escolar. Buenos Aires: Unesco.

Touraine, A. (1997). ¿Podremos vivir juntos? Iguales y diferentes. Buenos Aires: Fondo de Cultura Económica.

Weinstein, J. (2002). Educación e integración social: una visión desde la práctica de las políticas educativas. En Fosis (eds.) Hoy es mi tiempo. Una ventana a la esperanza. Santiago de Chile: Fondo de Solidaridad e Inversión Social.

Zemelman, H. (1998). Sujeto: existencia y potencia. México, D. F.: Anthropos. 